INPLASY

PROTOCOL

To cite: Sun et al. Social cognition in children and adolescents with epilepsy: a meta-analysis. Inplasy protocol 202230011. doi:

10.37766/inplasy2022.3.0011

Received: 03 March 2022

Published: 03 March 2022

Corresponding author: Zhongquan Yi

yizhongquan@163.com

Author Affiliation:

Yancheng Third People's

Hospital

Support: Jiangsu commission of Health.

Review Stage at time of this submission: Data analysis.

Conflicts of interest:

None declared.

\section{Social cognition in children and adolescents with epilepsy: a meta-analysis}

Sun, Y1; Zhao, J2; Zhao, PW3; Zhang, H4; Zhong, JG5; Pan, PL6; Wang, GD7; Yi, ZQ; $;$ Xie, LL9?.

Review question / Objective: To our knowledge, no metaanalysis has summarized social cognitive performance in children and adolescents with epilepsy as independent groups. Therefore, we conducted this meta-analysis to examine differences between children and adolescents with epilepsy and HCs in terms of ToM and FER performance.

Condition being studied: Epilepsy is characterized by chronic, unprovoked and recurrent seizures, is the most frequent neurological disease in childhood and usually occurs in early development. Worldwide, it is estimated that approximately $\mathbf{5 0}$ million people suffer from the pain of epileptic seizures, with more than half of the cases beginning in childhood and adolescence. So a comprehensive understanding of children and adolescence with epilepsy has become the focus of widespread attention. Recently, a number of studies have assessed ToM or facial emotion recognition deficits in children and adolescents with epilepsy, but the conclusions are inconsistent. These inconsistent findings might be related to the small sample sizes in most studies. Additionally, the methods used to evaluate ToM or facial emotion recognition performance were varied across studies. A meta-analysis can increase statistical power, estimate the severity of these deficits, and help resolve conflicting findings.

INPLASY registration number: This protocol was registered with the International Platform of Registered Systematic Review and Meta-Analysis Protocols (INPLASY) on 03 March 2022 and was last updated on 03 March 2022 (registration number INPLASY202230011).

\section{INTRODUCTION}

Review question / Objective: To our knowledge, no meta-analysis has summarized social cognitive performance in children and adolescents with epilepsy as independent groups. Therefore, we conducted this meta-analysis to examine 
differences between children and adolescents with epilepsy and HCs in terms of ToM and FER performance.

Condition being studied: Epilepsy is characterized by chronic, unprovoked and recurrent seizures, is the most frequent neurological disease in childhood and usually occurs in early development. Worldwide, it is estimated that approximately $\mathbf{5 0}$ million people suffer from the pain of epileptic seizures, with more than half of the cases beginning in childhood and adolescence. So a comprehensive understanding of children and adolescence with epilepsy has become the focus of widespread attention. Recently, a number of studies have assessed ToM or facial emotion recognition deficits in children and adolescents with epilepsy, but the conclusions are inconsistent. These inconsistent findings might be related to the small sample sizes in most studies. Additionally, the methods used to evaluate ToM or facial emotion recognition performance were varied across studies. A meta-analysis can increase statistical power, estimate the severity of these deficits, and help resolve conflicting findings.

\section{METHODS}

Search strategy: A literature search of electronic databases (Web of Science, PubMed and Embase) was completed in November 20th, 2021. The key search parameters were: ["epileps"” or "seizure disorder"] AND ["social cognition" or "theory of mind" or "ToM" or "mentalizing" or "mentalizing" or "facial expression" or "facial emotion recognition" or "emotion"]. A backward citation search was also undertaken.

Participant or population: Children and adolescents with epilepsy. No restrictions on sex, ethnicity, education or economic status.

Intervention: Studies compare ToM and facial emotion recognition performance between children and adolescents with epilepsy and healthy controls.
Comparator: ToM and facial emotion recognition performance.

Study designs to be included: Case-control studies.

Eligibility criteria: First, duplicate items were removed. Subsequent primary screening of titles and abstracts were screened o remove ineligibility (i.e. literature review, abstracts, no mention of epilepsy, irrelevant measurements, or animal studies; see Figure 1). Finally, fulltext screening was performed to remove the unqualified ones.Studies were included if: 1) They were published in the English language and published in peer-reviewed journals; 2) They included standard measures to assess at least one domain of ToM or FER performance; 3) They had a research design that compared children and adolescents with epilepsy and HCs; 4) They presented adequate data to calculate effect sizes of ToM or FER; 5) They had to include individuals with epilepsy $<18$ years. Studies were excluded if: 1) They lacked HCs group; 2) The sample overlapped with another study with larger sample sizes; 3) They lacked comparisons of ToM or FER between children and adolescents with epilepsy and HC; 4) The sample size was less than 10.

Information sources: A literature search of electronic databases (Web of Science, PubMed and Embase) was completed in November 20th, 2021. A backward citation search was also undertaken.

Main outcome(s): The main results included Tom and facial emotion recognition tasks. In addition, the data used to calculate the effect amount and standard error of Tom / facial emotion recognition task were also included.

Additional outcome(s): Additional results included information on clinical symptoms of epilepsy.

Quality assessment / Risk of bias analysis: Based on the Newcastle-Ottawa Scale, a nine-star protocol was used to assess study quality. 
Strategy of data synthesis: For analyses, the Stata 15.0 software package with a random-effects model will be used. Hedges $\mathrm{g}$ and $95 \%$ confidence interval $(\mathrm{Cl})$ will be calculated as the index of effect size. The interpretation of Hedges is similar to Cohen d: 0.2 indicated a small effect, 0.5 indicated a medium effect, and 0.8 indicated a large effect.

Subgroup analysis: Subgroup analyses wille be performed to assess to investigate the impairment in different aspects of ToM tasks (including cognitive ToM and affective ToM) and individual ToM tasks (Strange stories, RMET, FPT, FBT, NEPSYII). Besides, considering that epileptic seizures are categorized by seizure onset into generalized or partial. Specific subgroup analyses will be performed to assess whether the defects of ToM and FER are related to the site of seizure focus (including focal epilepsy and generalized epilepsy).

Sensitivity analysis: We will conduct a sensitivity analysis to test the influence of each dataset, methodological quality, and the potential impacts of missing data on the pooled results. If publication bias was found, we will apply the trim-and-fill method to provide effect sizes adjusted for publication bias.

Language: English.

Country(ies) involved: China.

Keywords: adolescents; children; epilepsy; social cognition; theory of mind; facial emotion recognition; meta-analysis.

Contributions of each author:

Author 1 - Yang Sun - The author drafted the manuscript.

Email: sunyang446508415@qq.com

Author 2 - Jing Zhao - The author provided statistical expertise.

Email: zhaojing23@163.com

Author 3 - PanWen Zhao - The author contributed to the development of the selection criteria, the risk of bias assessment strategy, and data extraction. Email: zhaopanwen8@163.com
Author 4 - Hui Zhang - The author contributed to the development of the selection criteria, the risk of bias assessment strategy, and data extraction.

Email: zhang598070@163.com

Author 5 - JianGuo Zhong - The author provided methodological support and software analysis guidance.

Email: yc3yjgzhong@163.com

Author 6 - PingLei Pan - The author provided methodological support and software analysis guidance.

Email: panpinglei@163.com

Author 7 - GenDi Wang - The author provided methodological support and software analysis guidance.

Email: wgd148888@sina.com

Author 8 - ZhongQuan Yi - The author contributed to conception, design, data analysis, and writing the manuscript.

Email: yizhongquan@163.com

Author 9 - LILI Xie - The author contributed to conception, design, data analysis, and writing the manuscript.

Email: 1019078626@qq.com 